\title{
Plant immunity: towards an integrated view of plant-pathogen interactions
}

Peter N. Dodds* and John P. Rathjen ${ }^{\ddagger}$

Abstract | Plants are engaged in a continuous co-evolutionary struggle for dominance with their pathogens. The outcomes of these interactions are of particular importance to human activities, as they can have dramatic effects on agricultural systems. The recent convergence of molecular studies of plant immunity and pathogen infection strategies is revealing an integrated picture of the plant-pathogen interaction from the perspective of both organisms. Plants have an amazing capacity to recognize pathogens through strategies involving both conserved and variable pathogen elicitors, and pathogens manipulate the defence response through secretion of virulence effector molecules. These insights suggest novel biotechnological approaches to crop protection.

\section{Elicitors}

Molecules that induce ('elicit') an immune defence response. In the context of this Review, this term is used to refer to both pathogen-associated molecular patterns (PAMPs) and effectors.

Pathogen-associated molecular patterns Any of a number of conserved, usually structural, molecules common to pathogen organisms.

${ }^{*}$ Commonwealth Scientific and Industrial Research Organisation (CSIRO), Division of Plant Industry, GPO BOX 1600, Canberra, Australian Capital Territory 2601, Australia.

${ }^{\ddagger}$ Research School of Biology, Australian National University, RN Robertson Building, Biology Place, Acton, Australian Capital Territory 0200, Australia. e-mails:peter.dodds@csiro.au: john.rathjen@anu.edu.au doi:10.1038/nrg2812

Published online 29 June 2010
Food security has become an issue of global importance, and major price spikes for staples such as rice and wheat have occurred in recent years. These price spikes are partly due to the impact of plant diseases, such as the spread of a new strain of the wheat stem rust pathogen from East Africa into the Middle East ${ }^{1}$. This has sparked an increased focus on improving approaches to crop protection. The most effective and environmentally sensitive approach to disease prevention involves breeding crop plants for resistance. Indeed, plant breeders have been using 'resistance' genes to control diseases in crop plants for almost 100 years, and the effectiveness of this strategy sparked early genetic studies that defined 'gene-for-gene' relationships between host resistance genes and pathogen virulence factors ${ }^{2}$. However, only through recent molecular studies has it become apparent that host resistance genes encode components of the plant immune system that confer the capacity to recognize and respond to specific pathogens. Plant immunity depends on cell-autonomous events; these events are related to the innate immune system in animals ${ }^{3}$ but plants have a much bigger recognition repertoire to compensate for their lack of an adaptive immune system. Ongoing research is revealing the recognition capacity of the plant immune system, and concurrent studies on pathogen biology are beginning to unravel how these organisms manipulate host immunity to cause disease. The recent convergence of these two fields has dramatically changed our perception of plant-pathogen interactions and is providing new approaches for crop protection.
Microbial plant pathogens almost always occupy extracellular niches. Despite this, the nutrients that enable pathogen growth are derived from host cells, and the host cytoplasm and organelles are important sites of molecular interaction. Plants have evolved two strategies to detect pathogen ${ }^{4,5}$ (FIG. 1). On the external face of the host cell, conserved microbial elicitors called pathogenassociated molecular patterns (PAMPs) are recognized by receptor proteins called pattern recognition receptors (PRRs) ${ }^{6}$. PAMPs are typically essential components of whole classes of pathogens, such as bacterial flagellin or fungal chitin. Plants also respond to endogenous molecules released by pathogen invasion, such as cell wall or cuticular fragments called danger-associated molecular patterns (DAMPs). Stimulation of PRRs leads to PAMP-triggered immunity (PTI). The second class of perception involves recognition by intracellular receptors of pathogen virulence molecules called effectors; this recognition induces effector-triggered immunity (ETI). This mode of recognition leads to co-evolutionary dynamics between the plant and pathogen that are quite different from PTI as, in stark contrast to PAMPs, effectors are characteristically variable and dispensable. Extreme diversification of ETI receptors and pathogen effectors both within and between species is the norm, whereas some PRR functions are conserved widely across families. Generally, PTI and ETI give rise to similar responses, although ETI is qualitatively stronger and faster and often involves a form of localized cell death called the hypersensitive response (HR). PTI is generally effective against non-adapted pathogens in a phenomenon called non-host resistance, 
Effectors

Proteins secreted by pathogens into host cells

to enhance infection.

Many of these function to

suppress PAMP-triggered

immunity responses.

\section{Extracellular recognition by PRRs}

PRRs have been reviewed recently ${ }^{7}$, so here we discuss some important principles and recent findings relating to key proteins in the process of recognition of

\begin{abstract}
Pattern recognition
receptors

Plasma membrane-localized

receptors that recognize

the presence of pathogen-

associated molecular

patterns (PAMPs) in the

extracellular environment.

PAMP-triggered immunity

The plant defence response

elicited by pathogen-

associated molecular

pattern (PAMP) recognition.

whereas ETI is active against adapted pathogens. However these relationships are not exclusive and depend on the elicitor molecules present in each infection.

Here, we provide an overview of the plant PTI and ETI systems, highlighting recent advances and identifying key gaps in our understanding of these processes. We consider the roles of PRRs in initial pathogen perception, our expanding knowledge of pathogen effectors and their roles in suppressing PTI responses, the nature of effector recognition and the downstream responses to pathogen perception. Finally, we discuss briefly how this knowledge is beginning to feed back into the agricultural context that originally spawned the study of plant immunity.
\end{abstract} extracellular pathogen molecules.

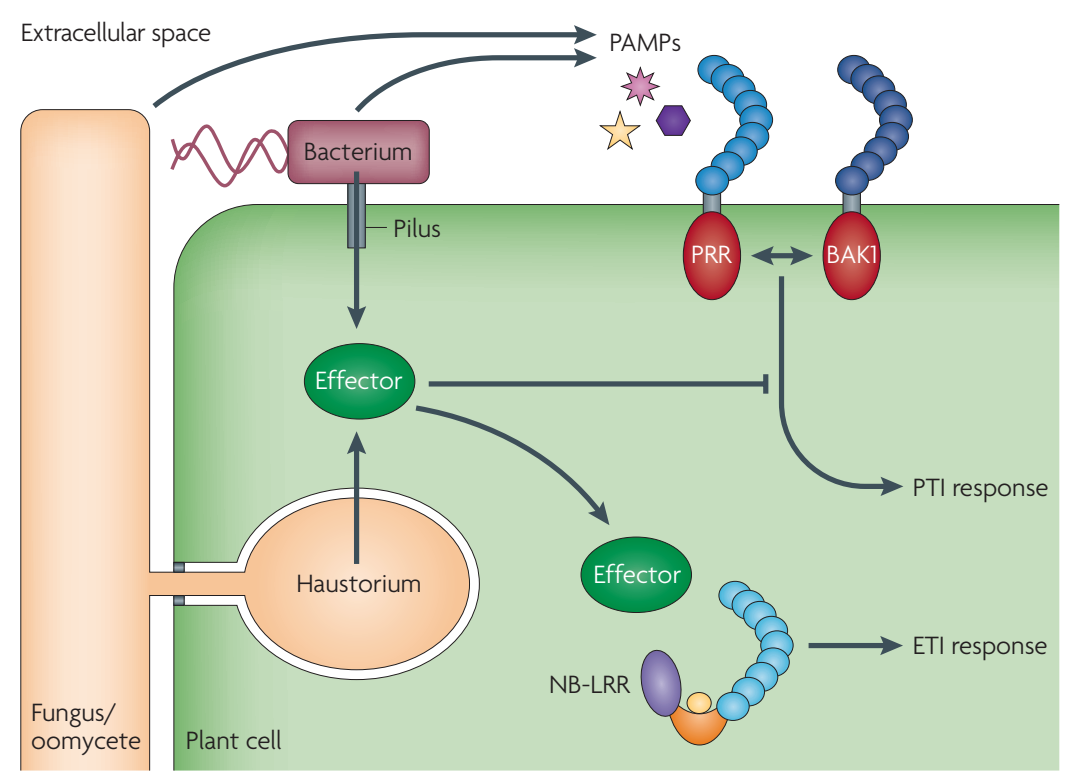

Figure 1 | The principles of plant immunity. Bacterial plant pathogens propagate exclusively in the extracellular spaces of plant issues. Most fungal and oomycete pathogens also extend their hyphae into this space, although many also form specialized feeding structures, known as haustoria, that penetrate host cell walls but not the plasma membrane. Other fungi extend invasive hyphae into plant cells, but again do not breach the host membrane. Molecules released from the pathogens into the extracellular spaces, such as lipopolysaccharides, flagellin and chitin (pathogenassociated molecular patterns (PAMPs)) are recognized by cell surface pattern recognition receptors (PRRs) and elicit PAMP-triggered immunity (PTI). PRRs generally consist of an extracellular leucine-rich repeat (LRR) domain (mid-blue), and an intracellular kinase domain (red). Many PRRs interact with the related protein BRASSINOSTEROID INSENSITIVE 1-ASSOCIATED KINASE 1 (BAK1) to initiate the PTI signalling pathway. Bacterial pathogens deliver effector proteins into the host cell by a type-III secretion pilus, whereas fungi and oomycetes deliver effectors from haustoria or other intracellular structures by an unknown mechanism. These intracellular effectors often act to suppress PTI. However, many are recognized by intracellular nucleotide-binding (NB)-LRR receptors, which induces effectortriggered immunity (ETI). NB-LRR proteins consist of a carboxyl-terminal LRR domain (light blue), a central NB domain (orange crescent) that binds ATP or ADP (yellow oval), and an amino-terminal Toll, interleukin-1 receptor, resistance protein (TIR) or coiled-coil (CC) domain (purple oval).
Pattern recognition receptors. Known PRRs fall into one of two receptor classes: transmembrane receptor kinases and transmembrane receptor-like proteins, the latter of which lack any apparent internal signalling domain ${ }^{7}$. Recent work has shown that endoplasmic reticulum quality-control mechanisms are crucial for PRR biogenesis (BOX 1). The receptor kinase gene family has undergone huge expansion in plants: for example, about 610 members are present in the Arabidopsis thaliana genome, and many of these are responsive to biotic stresses ${ }^{8}$. The receptor-like protein class has 57 members in A. thaliana ${ }^{9}$. The expansion of these families is in contrast to the situation in animals, which possess 12 Toll-like receptors that fulfil an equivalent role to PRRs in plants ${ }^{10}$.

The PAMPs recognized by plants are multifarious and include proteins, carbohydrates, lipids and small molecules, such as $\mathrm{ATP}^{6}$. Recognition of PAMPs is best understood in the case of the A. thaliana receptor kinase FLAGELLIN SENSING 2 (FLS2), which binds bacterial flagellin directly and then assembles an active signalling complex. Although the PAMP concept encompasses the idea that all PAMPs should be recognized by all species, this has been found to not always be the case, as perception of the bacterial elongation factor EF-Tu is apparently restricted to the Brassicaceae ${ }^{11}$. Similarly, the Xa21 receptor in rice provides race-specific resistance to the bacterial pathogen Xanthomonas oryzae, and was recently shown to act as a PRR for a novel sulphonated bacterial protein termed Ax21 (REF. 12).

$B A K 1$, a central regulator of PAMP-triggered immunity. Most known PRRs require the leucine-rich repeat (LRR) receptor kinase BRASSINOSTEROID INSENSITIVE 1-ASSOCIATED KINASE 1 (BAK1) for function $^{13,14}$ (FIG. 2). An exception is the fungal chitin receptor CHITIN ELICITOR RECEPTOR KINASE 1 $(\underline{\mathrm{CERK}} 1)^{15,16}$, which also responds to an unknown bacterial PAMP ${ }^{17}$. BAK1 is part of a family of five somatic embryogenesis receptor kinase (SERK) members and is also known as SERK3. It is not yet known whether other SERK family members have redundant roles in immune signalling. BAK1 does not have a direct role in elicitor perception, but FLS2 rapidly forms a complex with BAK1 after elicitation. This interaction results in phosphorylation of both proteins, which peaks 30-60 seconds after elicitor treatment ${ }^{18}$. BAK1 also has a role in the perception of other elicitors, probably also through heterodimerization with PRRs in the LRR-receptor kinase family.

As such, BAK1 is a central regulator of plant immunity and consequently the target of several pathogen virulence effector molecules ${ }^{19}$ (see below). Despite this, A. thaliana plants containing a null mutation in the bak1 gene are actually marginally more resistant to biotrophic pathogens, although they are slightly more susceptible to necrotrophic pathogen $s^{20}$. These phenotypes may be related to a deregulated cell death phenotype that has been described in the bak1 mutants ${ }^{20,21}$. 


\section{Box 1 | Pattern recognition receptor biogenesis}

Most eukaryotic membrane proteins undergo quality control during folding and maturation in the endoplasmic reticulum (ER), a process termed ER-QC ${ }^{114}$. A number of recent studies show that the biogenesis of a pattern recognition receptor (PRR), the EF-Tu receptor (EFR), is regulated by this mechanism ${ }^{115-119}$. After secretion into the ER, proteins are modified at glycosylable Asn residues by an oligosaccharyltransferase complex, which covalently attaches a complex polysaccharide containing three terminal glucose residues. The glucose moieties are subsequently trimmed by glucosidases I and II. A single glucose residue is added back by UDP-glucose:glycoprotein glucosyltransferase (UGGT) near regions of protein disorder. Monoglucosylated proteins interact with the lectins calnexin (CNX) or calreticulin (CRT) to retain misfolded substrates in the ER. In this way, UGGT acts as a folding sensor, and glycosylation is intimately related to protein maturation. Terminally misfolded proteins are degraded.

Another ER folding pathway is based on the chaperone BiP (a form of heat shock protein $70(\mathrm{Hsp} 70)$ ). Unfolded proteins undergo cycles of BiP binding and release, which is regulated by $\mathrm{Hsp} 40$ co-chaperones containing J domains (for example, the ERdj protein). Forward genetic screens showed that Arabidopsis thaliana genes encoding glucosidase II, UGGT, CRT3, ERdj3B and ERD2b are required for EFR function and accumulation. In addition, STT3A, a subunit of the oligosaccharyltransferase complex, was necessary for EFR biogenesis. Finally, STROMALDERIVED FACTOR 2 (SDF2) resides in a protein complex with ERdj3B and BiP, and was also required for EFR maturation. Plants with mutations in these genes are generally more susceptible to pathogens, indicating that EFR is not the only immune protein that is governed by ER-QC. However, neither FLAGELLIN SENSING 2 (FLS2) nor CHITIN ELICITOR RECEPTOR KINASE 1 (CERK1) function is significantly affected in these mutants.

Effector-triggered immunity The plant defence response elicited by effector recognition.

\section{Biotrophic}

Biotrophic pathogens propagate in living plant tissue and generally do not cause necrosis as a result of infection. They use various means, such as haustoria production, to extract nutrients from host cells.

\section{Necrotrophic}

Necrotrophic pathogens actively induce necrosis in infected tissues, often through the production of toxins, and obtain nutrients from the dead host tissue.

Type-III secretion system A syringe-like structure produced by many plant and animal pathogen bacteria that allows direct secretion of effector proteins from the bacterial cytoplasm into host cells.
One potential regulator of the FLS2-BAK1 complex is the cytoplasmic protein kinase BOTRYTISINDUCED KINASE 1 (BIK1). BIK1 was identified as a potential regulator because $b i k 1$ is upregulated after pathogen or elicitor treatment of $A$. thaliana leaves ${ }^{22}$. BIK1 interacts with both FLS2 and BAK1 before elicitation and seems to dissociate from the complex after elicitation. In vitro, BAK1 phosphorylates BIK1 and BIK1 phosphorylates both FLS2 and BAK1. In vivo, BIK1 becomes phosphorylated 5-10 min after treatment with flagellin ${ }^{23}$; this phosphorylation peaks after the FLS2-BAK1 phosphorylation. Confusingly, bik1 mutant $A$. thaliana plants are more resistant to Pseudomonas syringae infection than wild-type A. thaliana plants ${ }^{22}$ as a result of them overproducing the defence hormone salicylic acid (SA), but they are also more susceptible to infection with the necrotrophic fungal pathogen Botrytis cinerea. Despite this, deficiencies in FLS2-mediated immune responses could be measured in these plants ${ }^{23}$. These contrasting results make it difficult to ascribe a clear function to BIK1 in plant immunity, and further studies will be required.

\section{Virulence activities of pathogen effectors}

Successful pathogens are able to suppress PTI responses and thereby multiply and cause disease. They achieve suppression through the deployment of 'effector' proteins. Studies of bacterial phytopathogens have provided most of our understanding of effector strategies and mechanisms. Individual phytopathogen strains encode 20-30 effectors, which are highly regulated and secreted directly into the host cytoplasm by a dedicated needle structure, the type-III secretion system (TTSS) ${ }^{24}$. The repertoire of individual effectors varies dramatically among closely related bacterial strains, and effectors themselves act redundantly and are apparently interchangeable ${ }^{25}$; examples of such effectors are discussed below. Many effectors interfere directly with PTI responses ${ }^{26}$, and bacterial mutants that lack the TTSS system are non-pathogenic. Interestingly, a number of examples show that transgenic overexpression of an individual type-III effector in the host plant restores the ability of such bacterial mutants to grow $^{27,28}$, suggesting that bacterial pathogenicity only requires suppression of PTI. However, contributions of as yet undefined mechanisms to other processes, such as nutrient acquisition, cannot be excluded.

Bacterial effector functions. Bacterial effectors have molecular or enzymatic activities that specify both their ability to modify host targets and their intracellular recognition by ETI receptors ${ }^{29}$ (see below). The redundancy among effectors is illustrated by the unrelated P. syringae effectors AvrPto and AvrPtoB, which both target the FLS2-BAK1 complex. Although the models for how suppression works conflict in molecular detail ${ }^{19,30,31}$, it is generally accepted that AvrPtoB uses a dual strategy for kinase suppression: its aminoterminal kinase-targeting domain is sufficient to suppress flagellin responses, and its carboxy-terminal E3 ligase domain can tag interacting kinase proteins with ubiquitin to direct them for degradation ${ }^{32,33}$. AvrPtoB is known to target five host kinases of the Pto/interleukin receptor-associated kinase (IRAK) class $^{32}$, but because this clade is hugely expanded in plants ${ }^{8}$, there are probably many more such targets. Likewise, AvrPto suppresses multiple PRR receptor kinases, perhaps by acting as a kinase inhibitor ${ }^{19,30,34}$. Overall, these effectors seem to be part of a bacterial strategy that targets host kinases nonspecifically.

A further example of overlapping effector functions involves the host protein RPM1-INTERACTING PROTEIN 4 (RIN4), which is targeted by the P. syringae effectors AvrB, AvrRPM1 and AvrRpt2 through different molecular strategies ${ }^{35,36}$. Recently, it was shown that the $P$. syringae effector HopF2 may also target RIN4 (REF. 37). Overexpression of HopF2 prevented degradation of RIN4 by the protease AvrRpt2 but did not alter the interactions of RIN4 with AvrRPM1 or AvrB. Bacteria that lack HopF2 have increased growth on lines that lack RIN4, suggesting that RIN4 could indeed be a target for virulence, but an indirect cause for this observation was not ruled out. RIN4 is a negative regulator of both PTI and $\mathrm{ETI}^{28,38}$, and also interacts with the plasma membrane $\mathrm{H}^{+}$-ATPases AHA1 and AHA2 to enhance stomatal opening ${ }^{39}$, a key event during bacterial pathogenicity on leaves. Thus it is not clear how targeting of RIN4 by multiple effectors would enhance bacterial virulence, as disruption of RIN4 should actually restrict pathogenicity. However, the number of effectors involved in this process is consistent with RIN4 being an important virulence target. 
a

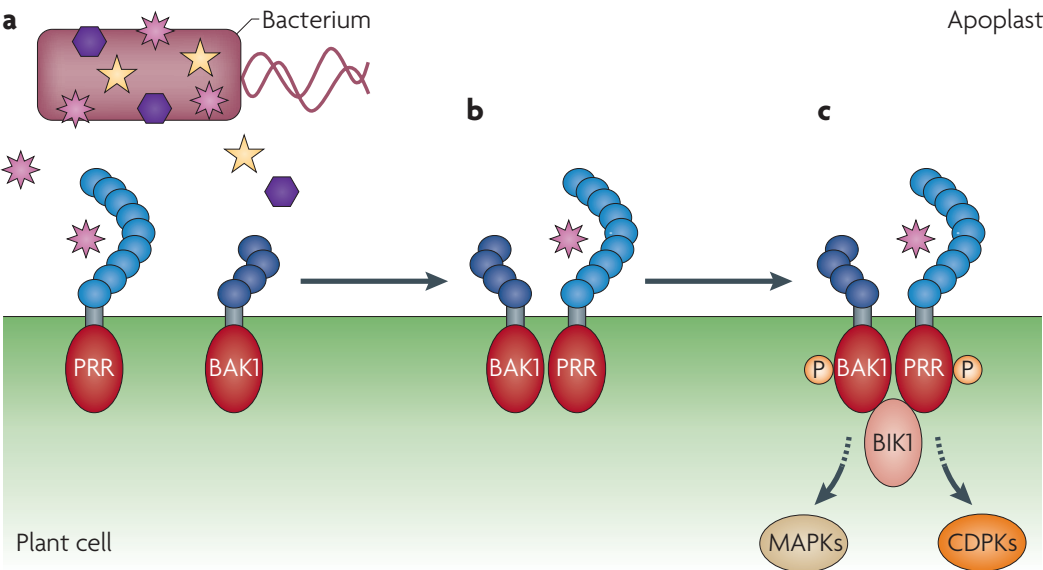

Figure 2 | Formation of active pattern recognition receptor complexes. a Infectious pathogens, such as bacteria, shed pathogen-associated molecular patterns (PAMPs; pink, yellow and purple shapes) into the apoplast, where they are recognized by specific pattern recognition receptors (PRRs). b| Immediately after ligand binding, the PRR forms an active complex with BRASSINOSTEROID

INSENSITIVE 1-ASSOCIATED KINASE 1 (BAK1). $\mathbf{c |}$ This results in transphosphorylation (indicated by P) of the respective kinase domains of the PRR and BAK1. Signalling via this active complex can be mediated directly by BOTRYTIS-INDUCED KINASE 1 (BIK1), or by mitogen-activated protein kinases (MAPKs) or calcium-dependent protein kinases (CDPKs). This is a generalized model that is based on FLAGELLIN SENSING 2 (FLS2), the receptor for bacterial flagellin.

\section{Haustoria}

(sing. haustorium.) Specialized structures produced by some fungal and oomycete pathogens. Haustoria extend through the plant cell wall and expand in the host cell. They remain surrounded by a host-derived membrane and hence are topologically extracellular and separated from the host cytoplasm.

Hemibiotrophic Hemibiotrophic pathogens incorporate aspects of both biotrophic and necrotrophic infection strategies. Often this involves an initial biotrophic infection phase during which the pathogen spreads in host tissue followed by a necrotrophic phase during which host cell death is induced.

NB-LRR proteins A class of intracellular receptor proteins containing nucleotide-binding (NB) and leucine-rich repeat (LRR) domains that recognize specific pathogen effectors.
It is important to note that not all effectors target PTI. One example of an alternative bacterial effector strategy is given by the transcription activator-like (TAL) effectors of Xanthomonas spp., which are transcription factors that induce the expression of specific host genes, some of which contribute to symptom development ${ }^{40}$. Unlike AvrPto and AvrPtoB in Pseudomonas spp., TAL effectors do not seem to act redundantly because several of them are essential for virulence. They interact specifically with a site in the target gene promoters through a central tandem repeat region that forms a DNA-binding domain ${ }^{41-43}$. Strikingly, two hypervariable amino acid residues in each repeat specify interaction with a characteristic nucleotide in the effector recognition site. Thus, the nucleotide sequence of the target DNA can be predicted by the amino acid sequence of the tandem repeat domain. Biotechnologically this is significant because it enables precise modification of gene expression in vivo, including turning this system against Xanthomonas spp. by engineering AvrBs3-responsive elements (known as UPA sites), upstream of active resistance genes ${ }^{44}$. In nature, this strategy has been pre-empted in some plant species: target sites for certain TAL effectors have been incorporated upstream of the resistance genes $B s 3$ and Xa27 in pepper and rice, respectively ${ }^{45,46}$.

Eukaryotic effectors. Data on eukaryotic effectors and their functions are sparse in comparison with data on bacterial effectors. Both fungal and oomycete pathogens produce effectors that are secreted through the endomembrane system and are subsequently delivered into host cells by unknown mechanisms ${ }^{47,48}$. Oomycete effectors characteristically contain the internal motif
Arg-X-Leu-Arg (RXLR, in which X represents any amino acid), which is required for delivery into plant cells. Genome sequencing of Phytophthora infestans ${ }^{49}$, the Irish potato famine pathogen, revealed 563 RXLR effector genes. Seventy of these genes are under diversifying selection and only 16 share orthologues in the genomes of 2 other sequenced Phytophthora spp., which indicates that very strong selection processes act on these effectors. A further 196 effectors of a separate class (known as Crinkler proteins) are encoded by P. infestans. Such generalized identification of fungal effector genes has been restricted by the lack of conserved motifs to aid genome interrogation, but genome analysis of several fungal pathogens predicts that they have complex and diversified secretomes ${ }^{50,51}$. The massive expansion in eukaryotic effector repertoires relative to bacterial effector repertoires may suggest a requirement for more diverse effector functions by eukaryotic pathogens, possibly to support their more specialized nutrient acquisition strategies.

Some data support roles of $P$. infestans effectors in suppression of immunity ${ }^{52}$; for example, Avr3a suppresses elicitor-induced cell death through interaction with the host CMPG1 E3 ligase $^{53}$, but in general very little is known about effector functions in fungi or oomycetes. However, many other potential roles remain, such as establishment of the pathogenic niche through development of the haustoria feeding structures and manipulation of host cell death during the hemibiotrophic lifestyle.

Sedentary nematode pathogens of plants form prolonged associations with roots, in which they induce the formation of novel host structures, such as multinucleate giant cells, from which they feed using a specialized proboscis called a stylet. The stylet also delivers salivary secretions into host cells; proteomic analysis of saliva from one such species, Meloidogyne incognita, identified 486 potential effector proteins ${ }^{54}$. Ongoing genomics analyses of such species will identify many more and help in elucidating the pathogenic strategies of these fascinating organisms. In addition, viral pathogens encode specific suppressors of the small RNA pathway to prevent degradation of their genomes and/or abrogation of viral gene expression ${ }^{55}$.

Overall, our understanding of effector proteins and their host targets is at a very early stage. Sophisticated biochemical screens for host protein targets that interact with the diverse suites of pathogen effectors are likely to lead to the identification of important components of host defence mechanisms and teach us more about host immune pathways and pathogenicity strategies.

\section{Intracellular effector recognition}

ETI is the second pathogen-sensing mechanism in plants and is based on intracellular recognition of effector proteins $s^{4,5}$. Recognition events are mostly mediated by a class of receptor proteins that contain nucleotidebinding (NB) domains and LRRs (FIG. 1). Plant NB-LRR proteins confer resistance to diverse pathogens, including fungi, oomycetes, bacteria, viruses and insects. NB and LRR domains are also present in NOD-like immune 
a Direct

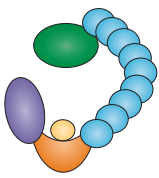

b Guard/decoy

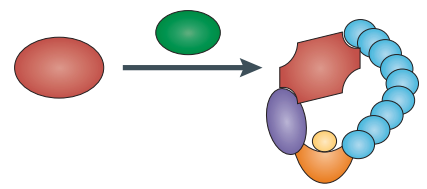

c Bait

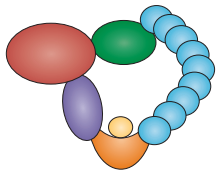

Figure 3 | Models of direct and indirect recognition. Plant nucleotide-binding (NB)-leucine-rich repeat (LRR) receptors can recognize pathogen effectors by either direct or indirect mechanisms. a | In direct recognition, the effector (green) triggers immune signalling by physically binding to the receptor (purple, orange, yellow and blue; see FIG. 1 for a description of the receptor). $\mathbf{b} \mid$ In the guard and decoy models, the effector modifies an accessory protein (red), which may be its virulence target (guard model) or a structural mimic of such a target (decoy model). The modified accessory protein is recognized by the NB-LRR receptor. c| Under the bait model, interaction of an effector with an accessory protein facilitates direct recognition by the NB-LRR receptor.

receptors (NLRs), which are involved in PAMP induction of innate immunity responses in animals ${ }^{3,56}$, and in the animal apoptotic factors apoptotic protease-activating factor 1 (APAF1) and cell death protein 4 (CED4). Many plant NB-LRR proteins also contain an N-terminal TIR (Toll, interleukin-1 receptor, resistance protein) domain related to the intracellular signalling domain of animal Toll-like receptors ${ }^{10}$. A second common class of NB-LRR proteins contain an $\mathrm{N}$-terminal domain with a coiledcoil (CC) domain, whereas others have no conserved $\mathrm{N}$-terminal region.

Direct and indirect recognition. NB-LRR proteins can recognize pathogen effectors either directly by physical association or indirectly through an accessory protein that is part of an NB-LRR protein complex (FIG. 3). In general, direct recognition has been demonstrated by yeast two-hybrid $(\mathrm{Y} 2 \mathrm{H})$ assays, in some cases supported by in vitro protein interaction assays. For example, the rice CC-NB-LRR Pi-ta protein binds to the Magnaporthe grisea effector AvrPita both in vitro and in $\mathrm{Y} 2 \mathrm{H}$ assays ${ }^{57}$. The flax TIR-NB-LRR L and M proteins also interact in $\mathrm{Y} 2 \mathrm{H}$ assays with the Melampsora lini fungal effectors AvrL567 and AvrM, respectively ${ }^{58-61}$. These pairs of receptor and effector proteins show evidence of strong diversifying selection and are characterized by high levels of sequence polymorphism between alleles in the host and pathogen populations, respectively, with these variants showing different recognition specificities. This is likely to be the result of antagonistic coevolution between the interacting components in the host and pathogen.

Indirect effector recognition has been observed in a number of cases. In the best-described models, the effector interaction is mediated by an accessory protein that is a pathogen virulence target or a structural mimic of one. The effector induces a change in the accessory protein that enables the accessory to be recognized by the NB-LRR protein ${ }^{62}$. This strategy neatly sidesteps the evolutionary advantage of the faster evolving pathogen, as the host takes advantage of the pathogen's virulence strategy to drive the recognition. Three conceptual models have been proposed to describe these mechanisms (FIG. 3). The 'guard' model postulates that NB-LRR proteins guard an accessory protein (or guardee) that is targeted and modified by pathogen effectors ${ }^{63}$. This model is exemplified by the A. thaliana RIN4 protein. RIN4 forms exclusive complexes with the NB-LRR proteins RPM1 and RESISTANCE TO PSEUDOMONAS SYRINGAE $2(\underline{\text { RPS2 }})^{36,64}$. Degradation of RIN4 by the protease effector AvrRpt2 de-represses RPS2, whereas AvrB or AvrRPM1-mediated phosphorylation of RIN4 activates RPM1 (REFS 35,36). Thus, modification of RIN4 by the effectors explains how an individual NB-LRR (in this case, RPM1) can recognize more than one effector.

However, the guard model postulates that RIN4 is a virulence target of the effectors, which is as yet unproven (see also above). Also, this model creates an evolutionary problem: RIN4 should evolve to avoid binding to the effector proteins in the absence of RPS2 and RPM1, but in their presence, selection will favour effector binding to promote recognition ${ }^{5}$. To solve this problem, the 'decoy' model was proposed ${ }^{62}$, in which duplication of the effector target gene or independent evolution of a target mimic could relax evolutionary constraints and allow the accessory protein to participate solely in effector perception. This situation is exemplified by the tomato NB-LRR protein Prf, which forms a complex with the accessory protein Pto kinase ${ }^{65}$. Pto kinase is closely related to the kinase domains of FLS2 and CERK1, which are targets of AvrPto and AvrPtoB ${ }^{32,66}$. Thus, Pto provides the recognition capability for Prf, and this drives diversification of the Pto family to broaden the spectrum of recognition capability ${ }^{67}$. In the decoy model, the accessory protein specializes in perception of the effector by the NB-LRR protein but has no other function. This fails to explain the requirement for Pto kinase activity in Prf activation ${ }^{68}$ and the clear role of RIN4 in defence responses. A further modification of the decoy concept is the bait-andswitch model ${ }^{69}$, which envisages a two-step recognition event. First, an effector interacts with the accessory 'bait' protein associated with an NB-LRR, and then a subsequent recognition event occurs between the effector and NB-LRR protein to trigger signalling. That is, the NB-LRR protein interacts with an effector target (the bait) to facilitate direct recognition of the pathogen effector, rather than recognizing the modified target as envisaged in the guard model.

It is important to remember that these models are generalizations based on limited specific examples, none of which is yet fully understood. Thus, although they are useful conceptual tools, they are unlikely to adequately describe all effector recognition events and can be restrictive. For instance, in addition to providing effector recognition, Pto seems to participate actively with Prf in a highly evolved co-regulatory relationship ${ }^{65,68}$. The massive diversity in effector and receptor biology suggests that many variations on these themes, and probably other novel recognition events, are likely to occur. For example, the Pto kinase phosphorylates the effector AvrPtoB, leading to inactivation of its intrinsic E3 ligase activity ${ }^{70}$; this is an intriguing and 
so far unique example of the host taking the bacteria's virulence strategy for its own. Interestingly, several examples have been described recently in which two different NB-LRR genes are required for recognition of specific effector proteins ${ }^{71-75}$, providing a further challenge for recognition models. The $A$. thaliana TIRNB-LRR genes RPS4 and RESISTANT TO RALSTONIA SOLANACEARUM 1 ( $\underline{R R S 1}$ ) are arranged in divergent tandem configuration within the major recognition gene complex MRC-J on chromosome 5 (REF. 76). RPS4 confers immunity to $P$. syringae through recognition of the effector AvrRps4, and RRS1 recognizes the Ralstonia solanacearum effector PopP2; new data show that both of these genes need to be expressed together for recognition of these effectors and for resistance to the fungus Colletotrichum higginsianum ${ }^{71}$. Genetic data suggest that the encoded proteins act in the same pathway, potentially as members of a protein heterocomplex. There are nine other examples of coordinate NB-LRR gene arrangement in the $A$. thaliana genome, and numerous other examples of NB-LRR genes working together have been described in various species. How these proteins function together remains unknown.

NB-LRR activation. One of the remaining challenges is to understand how effector recognition leads to NB-LRR activation, and whether the activation mechanisms are the same for different recognition systems. Broadly, the NB-LRR is a conserved multidomain switch that translates diverse direct or indirect pathogen signals into a general immune response ${ }^{69}$. Numerous genetic studies have shown that the LRR domain often controls recognition specificity ${ }^{77-80}$, with the implication that the LRR mediates effector interaction in these systems. However, these studies have necessarily been conducted on NB-LRRs that belong to diversified families, including some that are known to directly interact with their cognate effectors. By contrast, LRR domains of NB-LRRs that participate in indirect recognition are often conserved, and it is not clear what part the LRR domain plays in these cases. It is possible that direct and indirect recognition mechanisms involve fundamentally different NB-LRR activation processes.

In the absence of an effector trigger, NB-LRR proteins are maintained in a restrained conformation. In some indirect recognition systems, negative regulation of the NB-LRR by an accessory protein is released by effectors, and this is sufficient for activation of $\mathrm{ETI}^{81}$. This constitutes a simple paradigm that may occur widely. In other cases, the NB-LRR is autoinhibited ${ }^{75}$; that is, intramolecular interactions hold the protein in an inactive conformation until disrupted by the presence of the effector. This may be a general feature of direct recognition events. Nucleotide binding by the $\mathrm{NB}$ domain seems to be crucial for the function of all plant NB-LRR proteins ${ }^{75}$, and signal activation may involve an exchange of ATP and ADP in the binding site ${ }^{82}$. Biochemical analysis of NB-LRR proteins and their complexes has proven difficult but is crucial to advance our understanding of these complex activation events.
Animal NB-containing proteins, such as NLRs and the apoptotic factors APAF1 and CED4, self-oligomerize through their centrally located NB domain after activation, thereby forming an active signalling platform ${ }^{83}$. In this state, an N-terminal interaction domain (such as a caspase recruitment domain (CARD), pyrin domain or baculovirus inhibitor (BIR) domain) is made accessible for signalling adaptor proteins, which initiate the downstream signalling pathways leading to inflammatory response or apoptosis ${ }^{84}$. Similarly, the tobacco $\mathrm{N}$ protein oligomerizes in the presence of p50; oligomerization is dependent on a functional NB domain and also seems to involve the $\mathrm{N}$-terminal TIR domain ${ }^{85}$. Interestingly, tomato Prf exists in an oligomeric complex before stimulation with AvrPto or AvrPtoB ${ }^{67}$.

Similar to the N-terminal domains of mammalian NOD proteins, there is evidence that the TIR domain provides the downstream signalling capability for plant TIR-NB-LRR proteins. For instance, deletion or point mutations of the TIR domain from the tobacco N protein block HR induction downstream of the oligomerization event ${ }^{85}$. Furthermore, overexpression of the isolated TIR domains of several TIR-NB-LRR proteins is sufficient to trigger an $\mathrm{HR}^{86,87}$. The TIR domains of Toll-like receptors are activated by dimerization triggered by extracellular PAMP recognition ${ }^{10}$, so it is possible that effector-induced $\mathrm{R}$ protein oligomerization enables TIR activation through induced proximity. For some non-TIR NB-LRRs, overexpression of the CC-NBARC fragments can trigger plant defence signalling, whereas the CC domains alone do not ${ }^{88-90}$. In tobacco, the N-terminal portion of the NB domain of the $\mathrm{Rx}$ protein (which confers resistance to potato virus $\mathrm{X}$ ) is sufficient to induce cell death ${ }^{89}$.

\section{Signalling pathways and downstream responses} A number of cellular events associated with both PTI and ETI are known, essentially as correlative phenomena. These include a rapid influx of calcium ions from external stores, a burst of active oxygen species, activation of mitogen-activated protein kinases (MAPKs), reprogramming of gene expression, deposition of callosic cell wall appositions at sites of attempted infection and, often, localized cell death (HR). There is extensive overlap among the gene expression profiles elicited by most PAMPs 6 . PTI and ETI gene expression signatures are largely similar, suggesting that the responses are the same overall but vary in magnitude ${ }^{91}$. One of the big gaps in our understanding of plant immunity is in the signalling pathways that operate immediately downstream of PRR and NB-LRR protein activation. Genetic screens have had very limited success in identifying signalling components, and the components of these pathways remain mostly elusive. Several of the partially understood pathways are described below.

Kinase signalling. One topic that has received a lot of attention is MAPK signalling. MAPK pathways are ubiquitous signal transduction components in eukaryotes and transfer signals from extracellular 
receptors to cellular responses. A MAPK cascade typically consists of a modular complex consisting of a MAPK kinase kinase (MAPKKK), which phosphorylates a MAPK kinase (MAPKK), which phosphorylates a MAPK. These pathways regulate the activity of various substrates, such as transcription factors and protein kinases.

Importantly, MAPK cascades have been implicated in both PTI and ETI $^{92}$. A putative MAPK cascade that acts downstream of flagellin perception has been characterized in $A$. thaliana. It comprises the MAPKKs MKK4 and MKK5 upstream of the MAPKs MPK3 and MPK6, and leads to activation of WRKYtype transcription factors. The cascade culminates in the expression of defence genes ${ }^{93}$. Congruently, constitutively active MKK4 and MKK5 confer resistance to infection by $P$. syringae in A. thaliana. Previously, the MAPKKK MEKK1 was thought to be part of this cascade ${ }^{93}$ but more recent evidence indicates that this is unlikely, as mekk1 mutant plants are not compromised in activation of MPK3 and MPK6 triggered by the flagellin peptide flg22 (REF. 94). Rather, MEKK1 seems to act at the apex of a cascade comprising MEKK1, MKK1, MKK2 and MPK4, which is also activated by flg22 treatment. MPK3 and MPK6 are also activated by other PAMPs ${ }^{6}$. MPK6 activates ethylene biosynthesis through phosphorylation of 1-AMINOCYCLOPROPANE-1-CARBOXYLIC ACID SYNTHASE (ACS6) on flg22 perception ${ }^{95}$. Moreover, ERF104, an ethylene response factor, is a known MPK6 substrate $^{96}$. The MPK6-ERF104 interaction is rapidly lost in response to flg22, presumably allowing the liberated ERF104 to access target genes and activate ethylene signalling during $\mathrm{PTI}^{96}$.

In a recent paper, Sheen and colleagues defined an alternative pathway based on activation of calciumdependent protein kinases (CDPKs) ${ }^{97}$. Using a functional genomics approach, they defined a subclade of $A$. thaliana CDPKs that are required for FLS2dependent immunity. This pathway acts mostly independently of the MAPK pathway, as judged by gene expression assays, but antagonistic and synergistic effects were also observed. This model is consistent with the observation that calcium channel inhibitors abrogate most immune responses elicited by microbe-associated molecular patterns (MAMPs) or effectors.

Effector-triggered immunity signalling. Most of the genes identified in genetic screens for suppressors of ETI are either genes specific to the recognition system used in the screen, such as recognition accessory proteins, or members of a chaperone complex required for the function of many NB-LRR proteins ${ }^{98}$. Only a couple of genuine signalling proteins have been identified. ENHANCED DISEASE SUSCEPTIBILITY 1 (EDS1) is required for signalling of all TIR-NB-LRRs tested to date, suggesting that it acts specifically in TIR domain signalling ${ }^{99}$. However, it is not clear what intermediaries connect EDS1 and TIR-NB-LRRs. Similarly, the integral plasma membrane protein NON-RACE-SPECIFIC DISEASE RESISTANCE 1 (NDR1) is required for signalling from some CC-NB-LRRs (which are all membrane associated), but again the connecting steps are unknown ${ }^{100}$. The lack of success with genetic screens for signalling components could suggest that there are few essential elements in ETI signalling, and there is a possibility that redundant signalling pathways operate in parallel. Biochemical approaches for identifying signalling components interacting with activated NB-LRR proteins will be necessary to uncover further steps in these pathways.

Despite the difficultly in identifying components, an interesting model for ETI signalling has been proposed recently. In this model, NB-LRRs relocate to the nucleus on activation and interact with nuclear factors to trigger changes in gene expression. For instance, the tobacco N protein, barley MLA10 protein and A. thaliana RPS4 proteins localize to both the cell cytoplasm and nucleus, and nuclear accumulation is required for their function ${ }^{101-103}$. However, only a small fraction of these NB-LRR proteins is present in the plant cell nucleus. It can also be difficult to distinguish interactions related to recognition from those related to signalling. For example, A. thaliana RRS1-R interacts with the Ralstonia solanacearum effector PopP2 in the nucleus ${ }^{104,105}$. In addition, although a putative nuclear localization signal is required for the function of full-length RPS4 protein, it is not present in the constitutively active TIR domain of RPS4 (REF. 87). To date, no signalling partners common to different NB-LRR proteins have been identified in the nucleus. It will be interesting to discover the extent to which nuclear localization explains the signalling activity of these examples, and whether this is a general feature of all NB-LRR receptors.

Downstream responses. Some of the downstream responses to ETI and PTI are better understood than the signalling pathways. The SA and jasmonic acid (JA)ethylene (ET) hormone pathways are important regulators of defence-gene expression ${ }^{106}$. These two pathways act antagonistically to some extent, with SA involved in resistance to biotrophic pathogens and JA-ET involved in responses to necrotrophic pathogens and chewing insects. However, although there are substantial differences in the gene expression outputs of these pathways, and several genes act as specific markers for the activation of either the SA or JA-ET pathways, there is also considerable overlap between them. Recently, Tsuda et al. ${ }^{107}$ found complex interactions between SA and JA-ET signalling in a detailed combinatorial study using multiple mutants blocked in different pathways. The SA and JA-ET pathways seemed to act synergistically in PTI to amplify the response. This may explain why many pathogen effectors are able to suppress PTI by interacting with different targets; because the signal itself is relatively weak, blocking just one component is sufficient to substantially perturb the response. However, the ETI response is stronger and involves redundant activities of SA and JA-ET pathways ${ }^{107}$. Thus, even in the absence of SA signalling, the JA-ET response contributes to maintaining a substantial level of pathogen resistance. These compensatory 


\section{Box 2 | Novel agricultural applications}

Plant breeders have long recognized the importance of resistance genes for preventing disease in crop plants. Many of these genes have now been found to encode effector-triggered immunity (ETI) receptors, and we know that pathogens can evolve to overcome these genes through loss or alteration of the effectors that are recognized. The careful deployment of resistance genes in crop plants, particularly by using multiple effective receptors in combination and by selecting target effectors that have crucial virulence functions, should allow more durable resistance.

Many nucleotide-binding (NB)-leucine-rich repeat (LRR) genes have now been cloned, and this can facilitate their application in agriculture either through conventional breeding approaches, in which the cloned sequences are used as molecular markers, or through transgenic means. Widespread genome sequencing of plant pathogens is now yielding long lists of effector proteins that could be recognized by plant immune receptors, and these can now be screened against wild relatives of crop plants to identify new sources of resistance. This approach has been useful already in identifying new sources of resistance to the potato blight pathogen Phytophthora infestans in wild potatoes ${ }^{120}$. Pathogenassociated molecular pattern (PAMP)-triggered immunity (PTI) receptors are typically not variable within species and thus have not contributed widely to traditional breeding efforts. However, the transfer of these receptors among species has tremendous potential to deliver durable resistance, as the recognition components are highly conserved among pathogens. Although pathogens that are adapted to a particular host plant may be adept at suppressing the pattern recognition receptors (PRRs) of that host, their effectors might not recognize PRRs from other host plants. For instance, the Arabidopsis thaliana EF-Tu receptor occurs only in the Brassicaceae family, and transfer of this gene into tomato provided good resistance against various bacterial pathogens ${ }^{121}$. interactions may simply result from the higher signal flux in ETI, and probably make this response more robust against pathogen interference.

Despite, or perhaps because of, the number of gene expression changes resulting from PTI and ETI activation, the key changes that result in prevention of pathogen growth are not clear in any disease system. Perhaps the many responses each have such minor effects that individual contributions are difficult to quantify, but it is also likely that different aspects of the response are effective against different types of pathogens. Some recent work is beginning to unravel specific responses with significant effects on pathogen invasion. For instance, the PENETRATION 2 (PEN2) and PEN3 proteins of A. thaliana are involved in preventing cellular penetration by powdery mildew fungi ${ }^{108,109}$. PEN2 is a hydrolytic enzyme that produces a glucosinylate compound from an inactive precursor and $\mathrm{PEN} 3$ is an $\mathrm{ABC}$ transporter that seems to be involved in secretion of this molecule at the site of fungal $\operatorname{attack}^{110,111}$. Although these activities may have a direct antimicrobial role, both proteins are also required for the deposition of callose at the infection site and encasement of powdery mildew haustoria, suggesting a more subtle regulatory role in blocking infection. The Lr34 gene of wheat, which has been widely used in agriculture because it confers broad spectrum resistance against leaf and stripe rust fungi as well as powdery mildew, was also recently shown to encode an $\mathrm{ABC}$ transporter ${ }^{112}$. RPW8 in A. thaliana is another protein that provides broad spectrum resistance to powdery mildew fungi. It is targeted to a host-derived membrane that surrounds the fungal infection structures and also acts to enhance the callosic encasement of the fungal haustorium ${ }^{113}$.

\section{Conclusions}

The current synthesis of plant-pathogen molecular interactions provides a strong conceptual framework for understanding how these organisms coexist. Plants have evolved innate immune systems that recognize the presence of potential pathogens and initiate effective defence responses, whereas successful pathogens have evolved effector proteins that can suppress host immune responses. Furthermore, effectors can themselves act as elicitors and can be disabled by the host. Overall, the pathogenic niche is highly evolved and carefully monitored by both participants.

Despite the advances in characterization of individual molecular interactions and their consequences, our understanding of host-pathogen molecular co-evolution is poorly developed. The dominant synthesis, as currently understood, invokes a molecular arms race. However, this area is in fact relatively unexplored and both hosts and pathogens have generally not evolved rapid mechanisms to generate massive diversity in pathogen elicitors or host receptors, respectively. It is crucially important for the deployment of existing and novel resistance genes in agriculture that we advance our knowledge in this area to aid predictions of how changes in selection parameters will affect the evolution of pathogens, at both microscale and population levels. This need for a better understanding of co-evolution is particularly true for breakthrough technologies, such as the deployment of new PAMP-recognition specificities in crop species (BOX 2).

Moreover, there are many fundamental molecular questions about which we are still ignorant, such as what are the distinct and common signalling components of PTI and ETI? How are NB-LRR proteins activated by effector recognition? What are the induced host components and compounds downstream of pathogen perception that effect immunity? What are the targets of effectors, and how does the deployment of these effectors maximize the pathogenic niche? And what are the effector delivery mechanisms of fungi and oomycetes? Widespread genome sequencing of both host and pathogen genomes will facilitate the identification of effector proteins, the genome-wide analysis of dynamic effector expression patterns and the identification, through proteomics and gene homology, of host target proteins. The immediate technological impact of next-generation sequencing will open up the study of important non-model host-pathogen systems, such as wheat rusts and the black sigatoka disease of banana. One promising avenue is to exploit the diversity of plant species to access useful pathogen receptors from sexually incompatible host plants, which will expand the resource of resistance genes that can be transferred into agricultural species. However, filling in many of the current gaps in knowledge will require the application of biochemical, structural and cell biology approaches to unravel the molecular events associated with receptor activation and downstream signalling pathways. 
Singh, R. P. et al. in Advances in Agronomy (ed. Sparks, D. L.) Vol. 98 Ch. 5, 271-309 (Elsevier, London, 2008)

2. Flor, H. H. Current status of gene-for-gene concept. Annu. Rev. Phytopathol. 9, 275-296 (1971).

3. Ausubel, F. M. Are innate immune signaling pathways in plants and animals conserved? Nature Immunol. $\mathbf{6}$, 973-979 (2005)

4. Chisholm, S. T., Coaker, G., Day, B. \& Staskawicz, B. J. Host-microbe interactions: shaping the evolution of the plant immune response. Cell 124, 803-814 (2006).

5. Jones, J. D. \& Dangl, J. L. The plant immune system. Nature 444, 323-329 (2006).

6. Boller, T. \& Felix, G. A renaissance of elicitors: perception of microbe-associated molecular patterns and danger signals by pattern-recognition receptors. Annu. Rev. Plant Biol. 60, 379-406 (2009).

7. Zipfel, C. Pattern-recognition receptors in plant innate immunity. Curr. Opin. Immunol. 20, 10-16 (2008).

8. Lehti-Shiu, M. D., Zou, C., Hanada, K. \& Shiu, S.-H. Evolutionary history and stress regulation of plant receptor-like kinase/pelle genes. Plant Physiol. 150 12-26 (2009).

9. Wang, G. et al. A genome-wide functional investigation into the roles of receptor-like proteins in Arabidopsis. Plant Physiol. 147, 503-517 (2008).

10. Gay, N. J. \& Gangloff, M. Structure and function of Toll receptors and their ligands. Annu. Rev. Biochem. 76 141-165 (2007)

11. Zipfel, C. et al. Perception of the bacterial PAMP EF-Tu by the receptor EFR restricts Agrobacterium-mediated transformation. Cell 125, 749-760 (2006).

12. Lee, S.-W. et al. A type I-secreted, sulfated peptide triggers XA21-mediated innate immunity. Science 326, 850-853 (2009).

Although most PRRs provide subtle protection, this paper shows that a single PAMP receptor can confer effective disease resistance to a bacterial pathogen.

13. Chinchilla, D et al. A flagellin-induced complex of the receptor FLS2 and BAK1 initiates plant defence. Nature 448, 497-500 (2007).

14. Heese, A. et al. The receptor-like kinase SERK3/BAK1 is a central regulator of innate immunity in plants. Proc. Natl Acad. Sci. USA 104, 12217-12222 (2007). This study identified of BAK1 as a central regulator of plant immunity.

15. Miya, A. et al. CERK1, a LysM receptor kinase, is essential for chitin elicitor signaling in Arabidopsis. Proc. Natl Acad. Sci. USA 104, 19613-19618 (2007)

16. Wan, J. et al. A LysM receptor-like kinase plays a critical role in chitin signaling and fungal resistance in Arabidopsis. Plant Cell 20, 471-481 (2008).

17. Gimenez-lbanez, S., Ntoukakis, V. \& Rathjen, J. The LysM receptor kinase CERK 1 mediates bacterial perception in Arabidopsis. Plant Signal. Behav. 4, 539-541 (2009).

18. Schulze, B. et al. Rapid heteromerization and phosphorylation of ligand-activated plant transmembrane receptors and their associated kinase BAK1. J. Biol. Chem. 285, 9444-9451 (2010)

19. Shan, L. et al. Bacterial effectors target the common signaling partner BAK 1 to disrupt multiple MAMP receptor-signaling complexes and impede plant immunity. Cell Host Microbe 4, 17-27 (2008).

20. Kemmerling, B. et al. The BRI1-associated kinase 1 , BAK 1, has a brassinolide-independent role in plant cell-death control. Curr. Biol. 17, 1116-1122 (2007)

21. He, K. et al. BAK1 and BKK1 regulate brassinosteroiddependent growth and brassinosteroid-independent cell-death pathways. Curr. Biol. 17, 1109-1115 (2007)

22. Veronese, P. et al. The membrane-anchored BOTRYTIS-INDUCED KINASE 1 plays distinct roles in Arabidopsis resistance to necrotrophic and biotrophic pathogens. Plant Cell 18, 257-273 (2006).

23. Lu, D. et al. A receptor-like cytoplasmic kinase, BIK1, associates with a flagellin receptor complex to initiate plant innate immunity. Proc. Natl Acad. Sci. USA 107 496-501 (2010)

24. Cunnac, S., Lindeberg, M. \& Collmer, A. Pseudomonas syringae type III secretion system effectors: repertoires in search of functions. Curr. Opin. Microbiol. 12, 53-60 (2009).

25. Kvitko, B. H. et al. Deletions in the repertoire of Pseudomonas syringae pv. tomato DC3000 type III secretion effector genes reveal functional overlap among effectors. PLoS Pathog. 5, e 1000388 (2009). A clear, genetic demonstration that bacterial effectors work redundantly. This explains why individual deletions of effector genes often have minor phenotypes.
26. Zhou, J.-M. \& Chai, J. Plant pathogenic bacterial type III effectors subdue host responses. Curr. Opin. Microbiol. 11, 179-185 (2008)

27. Hauck, P., Thilmony, R. \& He, S. Y. A Pseudomonas syringae type III effector suppresses cell wall-based extracellular defense in susceptible Arabidopsis plants. Proc. Natl Acad. Sci. USA 100, 8577-8582 (2003).

28. Kim, M. G. et al. Two Pseudomonas syringae type III effectors inhibit RIN4-regulated basal defense in Arabidopsis. Cell 121, 749-759 (2005).

29. Grant, S. R., Fisher, E. J., Chang, J. H., Mole, B. M. \& Dangl, J. L. Subterfuge and manipulation: type III effector proteins of phytopathogenic bacteria. Annu. Rev. Microbiol. 60, 425-449 (2006).

30. Xiang, T. et al. Pseudomonas syringae effector AvrPto blocks innate immunity by targeting receptor kinases. Curr. Biol. 18, 74-80 (2008).

This paper shows that the bacterial effector AvrPto targets receptor kinases, providing the intellectual basis for current decoy models of indirect pathogen recognition.

31. Gohre, V. et al. Plant pattern-recognition receptor FLS2 is directed for degradation by the bacterial ubiquitin ligase AvrPtoB. Curr. Biol. 18, 1824-1832 (2008)

32. Gimenez-Ibanez, S. et al. AvrPtoB targets the LysM receptor kinase CERK 1 to promote bacterial virulence on plants. Curr. Biol. 19, 423-429 (2009).

33. Rosebrock, T. R. et al. A bacterial E3 ubiquitin ligase targets a host protein kinase to disrupt plant immunity. Nature 448, 370-374 (2007).

34. Xing, W. et al. The structural basis for activation of plant immunity by bacterial effector protein AvrPto. Nature 449, 243-247 (2007).

35. Axtell, M. J. \& Staskawicz, B. J. Initiation of RPS2specified disease resistance in Arabidopsis is coupled to the AvrRpt2-directed elimination of RIN4. Cell 112, 369-377 (2003).

36. Mackey, D., Belkhadir, Y., Alonso, J. M., Ecker, J. R. \& Dangl, J. Arabidopsis RIN4 is a target of the type III virulence effector AvrRpt2 and modulates RPS2 mediated resistance. Cell 112, 379-389 (2003).

37. Wilton, M. et al. The type III effector HopF2Pto targets Arabidopsis RIN4 protein to promote Pseudomonas syringae virulence. Proc. Natl Acad. Sci. USA 107, 2349-2354 (2010).

38. Marathe, R. $\&$ Dinesh-Kumar, S. P. Plant defense: one post, multiple guards?! Mol. Cell 11, 284-286 (2003)

39. Liu, J. et al. RIN4 functions with plasma membrane $\mathrm{H}^{+}$-ATPases to regulate stomatal apertures during pathogen attack. PLoS Biol. 7, e 1000139 (2009).

40. Kay, S. \& Bonas, U. How Xanthomonas type III effectors manipulate the host plant. Curr. Opin. Microbiol. 12, 37-43 (2009).

41. Kay, S., Hahn, S., Marois, E., Hause, G. \& Bonas, U. A bacterial effector acts as a plant transcription factor and Induces a cell size regulator. Science $\mathbf{3 1 8}$ 648-651 (2007).

42. Boch, J et al. Breaking the code of DNA binding specificity of TAL-Type III effectors. Science 326, 1509-1512 (2009).

43. Moscou, M. J. \& Bogdanove, A. J. A simple cipher governs DNA recognition by TAL effectors. Science 326, 1501 (2009).

44. Römer, P., Recht, S. \& Lahaye, T. A single plant resistance gene promoter engineered to recognize multiple TAL effectors from disparate pathogens. Proc. Natl Acad. Sci. USA 106, 20526-2053 (2009)

45. Gu, K. et al. R. gene expression induced by a type-III effector triggers disease resistance in rice. Nature 435, 1122-1125 (2005)

46. Romer, P. et al. Plant pathogen recognition mediated by promoter activation of the pepper Bs3 resistance gene. Science 318, 645-648 (2007)

47. Kamoun, S. Groovy times: filamentous pathogen effectors revealed. Curr. Opin. Plant Biol. 10 358-365 (2007)

48. Panstruga, R. \& Dodds, P. N. Terrific protein traffic: the mystery of effector protein delivery by filamentous plant pathogens. Science 324, 748-750 (2009).

49. Haas, B. J. et al. Genome sequence and analysis of the Irish potato famine pathogen Phytophthora infestans. Nature 461, 393-398 (2009).

50. Dean, R. A. et al. The genome sequence of the rice blast fungus Magnaporthe grisea. Nature 434 980-986 (2005)

51. Kämper, J. et al. Insights from the genome of the biotrophic fungal plant pathogen Ustilago maydis. Nature 444, 97-101 (2006).
52. Hogenhout, S. A., Van der Hoorn, R. A. L., Terauchi, R. $\&$ Kamoun, S. Emerging concepts in effector biology of plant-associated organisms. Mol. Plant Microbe Interact. 22, 115-122 (2009).

53. Bos, J. I. et al. Phytophthora infestans effector AVR3a is essential for virulence and manipulates plant immunity by stabilizing host E3 ligase CMPG 1. Proc. Natl Acad. Sci. USA 107, 9909-9914 (2010).

54. Bellafiore, S. et al. Direct identification of the Meloidogyne incognita secretome reveals proteins with host cell reprogramming potential. PLoS Pathog. 4, e1000192 (2008)

55. Voinnet, O. RNA silencing as a plant immune system against viruses. Trends Genet. 17, 449-459 (2001).

56. Girardin, S. E., Philpott, D. J. \& Lemaitre, B. Sensing microbes by diverse hosts. Workshop on pattern recognition proteins and receptors. EMBO Rep. 4, 932-936 (2003).

57. Jia, Y., McAdams, S. A., Bryan, G. T., Hershey, H. P. \& Valent, B. Direct interaction of resistance gene and avirulence gene products confers rice blast resistance. EMBO J. 19, 4004-4014 (2000).

58. Catanzariti, A. M., Dodds, P. N., Lawrence, G. J., Ayliffe, M. A. \& Ellis, J. G. Haustorially expressed secreted proteins from flax rust are highly enriched for avirulence elicitors. Plant Cell 18, 243-256 (2006).

59. Dodds, P. N., Lawrence, G. J., Catanzariti, A. M., Ayliffe, M. A. \& Ellis, J. G. The Melampsora lini AvrL567 avirulence genes are expressed in haustoria and their products are recognized inside plant cells. Plant Cell 16, 755-768 (2004).

60. Catanzariti, A. M. et al. The AvrM effector from flax rust has a structured $C$-terminal domain and interacts directly with the M resistance protein. Mol. Plant Microbe Interact. 23, 49-57 (2010).

61. Dodds, P. N. et al. Direct protein interaction underlies gene-for-gene specificity and coevolution of the flax resistance genes and flax rust avirulence genes. Proc. Natl Acad. Sci. USA 103, 8888-8893 (2006). An elegant demonstration of how direct recognition of pathogen effectors works at the molecular level and drives antagonistic co-evolution.

62. van der Hoorn, R. A. \& Kamoun, S. From guard to decoy: a new model for perception of plant pathogen effectors. Plant Cell 20, 2009-2017 (2008).

63. Dangl, J. L. \& Jones, J. D. Plant pathogens and integrated defence responses to infection. Nature 411, 826-833 (2001).

64. Mackey, D., Holt, B. F., Wiig, A. \& Dangl, J. L. RIN4 interacts with Pseudomonas syringae type III effector molecules and is required for RPM1-mediated resistance in Arabidopsis. Cell 108, 743-754 (2002).

65. Mucyn, T. S. et al. The tomato NBARC-LRR protein Prf interacts with Pto kinase in vivo to regulate specific plant immunity. Plant Cell 18, 2792-2806 (2006)

66. Zipfel, C. \& Rathjen, J. P. Plant immunity: AvrPto targets the frontline. Curr. Biol. 18, R218-R220 (2008).

67. Gutierrez, J. R. et al. Prf immune complexes of tomato are oligomeric and contain multiple Pto-like kinases that diversify effector recognition. Plant J. 61 507-581 (2009)

68. Mucyn, T. S., Wu, A. J., Balmuth, A. L., Arasteh, J. M. \& Rathjen, J. P. Regulation of tomato Prf by Pto-like protein kinases. Mol. Plant Microbe Interact. 22 391-401 (2009)

69. Collier, S. M. \& Moffett, P. NB-LRRs work a "bait and switch' on pathogens. Trends Plant Sci. 14, 521-529 (2009).

70. Ntoukakis, V. et al. Host inhibition of a bacterial virulence effector triggers immunity to infection. Science 324, 784-787 (2009).

71. Narusaka, M. et al. RRS 1 and RPS4 provide a dual resistance-gene system against fungal and bacterial pathogens. Plant J. 60, 218-226 (2009). An as-yet-unique example showing how the host can inactivate a pathogen effector, leading to its recognition and host immunity.

72. Sinapidou, E. et al. Two TIR:NB:LRR genes are required to specify resistance to Peronospora parasitica isolate Cala2 in Arabidopsis. Plant J. 38 898-909 (2004)

73. Loutre, C. et al. Two different CC-NBS-LRR genes are required for $L$ r 10 -mediated leaf rust resistance in tetraploid and hexaploid wheat. Plant J. 60 1043-1054 (2009).

74. Lee, S. K. et al. Rice Pi5-mediated resistance to Magnaporthe oryzae requires the presence of two coil ed-coil-nucleotide-binding-leucine-rich repeat genes. Genetics 181, 1627-1638 (2009). 
75. Takken, F. L. \& Tameling, W. I. To nibble at plant resistance proteins. Science 324, 744-746 (2009).

76. Birker, D. et al. A locus conferring resistance to Colletotrichum higginsianum is shared by four geographically distinct Arabidopsis accessions. Plant J. 60, 602-613 (2009).

77. Dodds, P. N., Lawrence, G. J. \& Ellis, J. G. Six amino acid changes confined to the leucine-rich repeat $\beta$-strand $/ \beta$-turn motif determine the difference between the $\mathrm{P}$ and $\mathrm{P} 2$ rust resistance specificities in flax. Plant Cell 13, 163-178 (2001).

78. Ellis, J. G., Lawrence, G. J., Luck, J. E. \& Dodds, P. N. Identification of regions in alleles of the flax rust resistance gene $L$ that determine differences in gene-for-gene specificity. Plant Cell 11, 495-506 (1999).

79. Shen, Q. H. et al. Recognition specificity and RAR1/ SGT1 dependence in barley Mla disease resistance genes to the powdery mildew fungus. Plant Cell 15 732-744 (2003)

80. Rairdan, G. J. \& Moffett, P. Distinct domains in the $A R C$ region of the potato resistance protein $R x$ mediate LRR binding and inhibition of activation Plant Cell 18, 2082-2093 (2006).

81. Belkhadir, Y., Nimchuk, Z., Hubert, D. A., Mackey, D. ¿ Dangl, J. L. Arabidopsis RIN4 negatively regulates disease resistance mediated by RPS 2 and RPM 1 downstream or independent of the NDR1 signal modulator and is not required for the virulence functions of bacterial type III effectors AvrRpt2 or AvrRpm1. Plant Cell 16, 2822-2835 (2004).

82. Tameling, W. I. et al. Mutations in the NB-ARC domain of $\mathrm{I}-2$ that impair ATP hydrolysis cause autoactivation Plant Physiol. 140, 1233-1245 (2006).

83. Inohara, N. et al. An induced proximity model for NF-kB activation in the Nod 1/RICK and RIP signaling pathways. J. Biol. Chem. 275, 27823-27831 (2000).

84. Shaw, M. H., Reimer, T., Kim, Y. G. \& Nunez, G. NOD-like receptors (NLRs): bona fide intracellular microbial sensors. Curr. Opin. Immunol. 20, 377-382 (2008).

85. Mestre, P. \& Baulcombe, D. C. Elicitor-mediated oligomerization of the tobacco $\mathrm{N}$ disease resistance protein. Plant Cell 18, 491-501 (2006).

86. Frost $D$ et al Tobacco transgenic for the flax rust resistance gene $L$ expresses allele-specific activation of defense responses. Mol. Plant Microbe Interact. 17, 224-232 (2004)

87. Swiderski, M. R., Birker, D. \& Jones, J. D. The TIR domain of TIR-NB-LRR resistance proteins is a signaling domain involved in cell death induction Mol. Plant Microbe Interact. 22, 157-165 (2009).

88. Bendahmane, A., Farnham, G., Moffett, P. \& Baulcombe, D. C. Constitutive gain-of-function mutants in a nucleotide binding site-leucine rich repeat protein encoded at the Rx locus of potato. Plant J. 32, 195-204 (2002).

89. Rairdan, G. J. et al. The coiled-coil and nucleotide binding domains of the potato Rx disease resistance protein function in pathogen recognition and signaling. Plant Cell 20, 739-751 (2008).

90. Tao, Y., Yuan, F., Leister, R. T., Ausubel, F. M. \& Katagiri, F. Mutational analysis of the Arabidopsis nucleotide binding site-leucine-rich repeat resistance gene RPS2. Plant Cell 12, 2541-2554 (2000).

91. Tao, Y. et al. Quantitative nature of Arabidopsis responses during compatible and incompatible interactions with the bacterial pathogen Pseudomonas syringae. Plant Cell 15, 317-330 (2003).

92. Pitzschke, A. Schikora, A \& Hirt, H. MAPK cascade signalling networks in plant defence. Curr. Opin. Plant Biol. 12, 421-426 (2009).
93. Asai, T. et al. MAP kinase signalling cascade in Arabidopsis innate immunity. Nature 415, 977-983 (2002)

94. Suarez-Rodriguez, M. C. et al. MEKK1 is required for flg22-induced MPK4 activation in Arabidopsis plants. Plant Physiol. 143, 661-669 (2007).

95. Liu, Y. \& Zhang, S. Phosphorylation of 1-aminocyclopropane-1-carboxylic acid synthase by MPK6, a stress-responsive mitogen-activated protein kinase, induces ethylene biosynthesis in Arabidopsis. Plant Cell 16, 3386-3399 (2004).

96. Bethke, G. et al. Flg22 regulates the release of an ethylene response factor substrate from MAP kinase 6 in Arabidopsis thaliana via ethylene signaling. Proc. Natl Acad. Sci. USA 106, 8067-8072 (2009).

97. Boudsocq, M. et al. Differential innate immune signalling via $\mathrm{Ca}^{2+}$ sensor protein kinases. Nature 464, 418-422 (2010)

98. Shirasu, K. \& Schulze-Lefert, P. Complex formation, promiscuity and multi-functionality: protein interactions in disease-resistance pathways. Trends Plant Sci. 8, 252-258 (2003).

99. Wiermer, M., Feys, B. J. \& Parker, J. E. Plant immunity the EDS1 regulatory node. Curr. Opin. Plant Biol. $\mathbf{8}$, 383-389 (2005)

100. Day, B., Dahlbeck, D. \& Staskawicz, B. J. NDR1 interaction with RIN4 mediates the differential activation of multiple disease resistance pathways in Arabidopsis. Plant Cell 18, 2782-2791 (2006)

101. Burch-Smith, T. M et al. A novel role for the TIR domain in association with pathogen-derived elicitors. PLoS Biol. 5, e68 (2007)

102. Shen, Q. H. et al. Nuclear activity of MLA immune receptors links isolate-specific and basal disease resistance responses. Science 315, 1098-1103 (2007).

103. Wirthmueller, L., Zhang, Y., Jones, J. D. \& Parker, J. E. Nuclear accumulation of the Arabidopsis immune receptor RPS4 is necessary for triggering EDS1dependent defense. Curr. Biol. 17, 2023-2029 (2007).

The key paper underlying the hypothesis that an active fraction of plant NB-LRR proteins resides in the plant cell nucleus.

104. Deslandes, L. et al. Physical interaction between RRS 1-R, a protein conferring resistance to bacterial wilt, and PopP2, a type III effector targeted to the plant nucleus. Proc. Natl Acad. Sci. USA 100 8024-8029 (2003)

105. Bernoux, M. et al. RD19, an Arabidopsis cysteine protease required for RRS1-R-mediated resistance, is relocalized to the nucleus by the Ralstonia solanacearum PopP2 effector. Plant Cell 20 2252-2264 (2008)

106. Bari, R. \& Jones, J. D. Role of plant hormones in plant defence responses. Plant Mol. Biol. 69, 473-488 (2009).

107. Tsuda, K., Sato, M., Stoddard, T., Glazebrook, J. \& Katagiri, F. Network properties of robust immunity in plants. PLoS Genet. 5, e1000772 (2009). A network approach to plant immunity shows complex interactions between defence hormone signalling pathways acting in both PTI and ETI.

108. Lipka, V. et al. Pre- and postinvasion defenses both contribute to nonhost resistance in Arabidopsis. Science 310, 1180-1183 (2005).

109. Stein, M. et al. Arabidopsis PEN3/PDR8, an ATP binding cassette transporter, contributes to nonhost resistance to inappropriate pathogens that enter by direct penetration. Plant Cell 18, 731-746 (2006).

110. Bednarek, P. et al. A glucosinolate metabolism pathway in living plant cells mediates broad-spectrum antifungal defense. Science 323, 101-106 (2009)
111 Clay, N. K., Adio, A. M., Denoux, C., Jander, G. \& Ausubel, F. M. Glucosinolate metabolites required for an Arabidopsis innate immune response. Science 323, 95-101 (2009).

112. Krattinger, S. G. et al. A putative ABC transporter confers durable resistance to multiple fungal pathogen in wheat. Science 323, 1360-1363 (2009). The first cloning of a broad spectrum resistance gene in wheat that is active against rusts and mildews. This study opened the way for the genetic manipulation of crop cultivars.

113. Wang, W., Wen, Y., Berkey, R. \& Xiao, S Specific targeting of the Arabidopsis resistance protein RPW8.2 to the interfacial membrane encasing the fungal Haustorium renders broad-spectrum resistance to powdery mildew. Plant Cell 21, 2898-2913 (2009).

114. Anelli, T. \& Sitia, R. Protein quality control in the early secretory pathway. EMBO J. 27, 315-327 (2008).

115. Häweker, H. et al. Pattern recognition receptors require $\mathrm{N}$-glycosylation to mediate plant immunity. J. Biol. Chem. 285, 4629-4636 (2010).

116. Li, J. et al. Specific ER quality control components required for biogenesis of the plant innate immune receptor EFR. Proc. Natl Acad. Sci. USA 106, 15973-15978 (2009).

117. Lu, X. et al. Uncoupling of sustained MAMP receptor signaling from early outputs in an Arabidopsis endoplasmic reticulum glucosidase II allele. Proc. Nat Acad. Sci. USA 106, 22522-22527 (2009).

118. Nekrasov, V. et al. Control of the pattern-recognition receptor EFR by an ER protein complex in plant immunity. EMBO J. 28, 3428-3438 (2009).

119. Saijo, Y. et al. Receptor quality control in the endoplasmic reticulum for plant innate immunity. EMBO J. 28, 3439-3449 (2009).

120. Vleeshouwers, V. G. A. A. et al. Effector genomics accelerates discovery and functional profiling of potato disease resistance and Phytophthora infestans avirulence genes. PLOS ONE 3, e2875 (2008)

121. Lacombe, S et al. Interfamily transfer of a plant patternrecognition receptor confers broad-spectrum bacterial resistance. Nature Biotech. 28, 365-369 (2010). A groundbreaking paper showing the potential for interfamily transfer of PRRs to provide broad spectrum disease protection in crop species.

\section{Acknowledgements}

We apologize to those authors whose work could not be cited owing to space limitations. J.P.R. is an Australian Research Council Future Fellow. Work in P.N.D.'s laboratory is funded by the Australian Research Council, the US National Institutes of Health and the Grains Research and Development Corporation. We thank J. Ellis and B. Staskawicz for helpful discussions.

Competing interests statement

The authors declare no competing financial interests.

DATABASES

AAIR: http://wwwarabidopsis.org

RPS4|RRS1

UniProtKB: http://www.uniprot.org

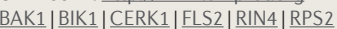

FURTHER INFORMATION

Peter N. Dodds' homepage:

http://www.csiro.au/people/Peter.Dodds.html

ohn P. Rathjen's homepage:

http://biology.anu.edu.au/Staff/Profiles/PS/Rathjen

ALL LINKS ARE ACTIVE IN THE ONLINE PDF 